\title{
An Auxiliary Decision-Making System for Electric Power Intelligent Customer Service Based on Hadoop
}

\author{
Shisong Wu $(\mathbb{D}$ and Zhaojie Dong \\ Digital Grid Research Institute, China Southern Power Grid, China \\ Correspondence should be addressed to Shisong Wu; wushisong@yccxip.com
}

Received 25 November 2021; Revised 30 December 2021; Accepted 3 January 2022; Published 19 January 2022

Academic Editor: Rahman Ali

Copyright (c) 2022 Shisong Wu and Zhaojie Dong. This is an open access article distributed under the Creative Commons Attribution License, which permits unrestricted use, distribution, and reproduction in any medium, provided the original work is properly cited.

\begin{abstract}
Aiming at the problems of low security, high occupancy rate, and long response time in the current power intelligent customer service assistant decision-making system, a power intelligent customer service assistant decision-making system based on the Hadoop big data framework is designed. By analyzing the Hadoop big data framework, according to the characteristics and core elements of the HDFS distributed file system, the MapReduce programming model, and the data mining algorithm, the basic process of power intelligent customer service assistance decision-making is established. We analyze the overall and functional requirements of the system, design the overall architecture and application architecture of the system, design the E-R diagram and table structure of the database according to the database design principle, and realize the design of power intelligent customer service auxiliary decision-making system based on Hadoop big data framework. The test results show that the proposed method has high system security and low system occupancy and can effectively shorten the system response time. The systems run more flawlessly as compared to the existing methods and give impressing results with lesser CPU utilization. The response time was recorded to be about 12.2 seconds for 1000 power intelligent customer servers, which is much lower than that of the competitors.
\end{abstract}

\section{Introduction}

With the continuous improvement of the national economy, the power industry has gradually become one of the important industries. In order to further enable users in the power industry to enjoy more comprehensive services, the service quality of the power industry must ensure a good state, so as to help power enterprises improve customer service quality, and to further improve the competitiveness of enterprises to a certain extent. At the same time, it is of certain significance to promote the stable development of power enterprises [1]. At this stage, due to the rapid development of the times, there are more challenging development requirements for the power system. Therefore, the power industry must take a long-term view, pay attention to and consider the development plan of the power industry, and accelerate the construction of power intelligent customer service system. At present, many power enterprises have begun to build intelligent customer service systems. By analyzing the operation status of power companies, it is verified that the key to the future development of the power industry is intelligence [2]. Accelerating the improvement of power customer systems, changing the traditional customer service mode, and diversifying customer service channels to a certain extent are the necessity of the steady development of power enterprises. Based on the traditional customer service mode, power enterprises generally only use the manual customer service hotline and physical store mode to provide customer service for power enterprises. Its customer service takes a long time and cannot adapt to the current development status and customer service needs [3]. In order to enable power users to obtain a faster and more efficient customer service mode, power enterprises must establish a power intelligent customer service auxiliary decision-making system. It can not only effectively reduce the work pressure of customer service personnel in power enterprises to a certain extent, but also effectively improve the work efficiency of customer service personnel in power enterprises 
to a certain extent, so as to further improve the quality of customer service in power enterprises.

At present, scholars in related fields have studied the power intelligent customer service decision-making and achieved some theoretical results. Reference [4] proposed a multicriteria decision support model for customer service quality evaluation. The multicriteria decision model is used to identify and classify the factors affecting after-sales service satisfaction. Put customer relationship marketing in the context and use cognitive mapping to determine the more relevant factors that affect customers' overall satisfaction with after-sales service. Reference [5] proposed the design of power system real-time decision emergency control system based on ultra-real-time simulation. According to the trajectory characteristics of the stability recovery process of enterprise owned power grid, a stability recovery criterion is proposed. A fast decision algorithm for frequency instability, and rotor angle instability, and branch overload with relatively low amount of calculation is introduced. The control strategy can be determined within a specific time after the fault is detected. This method can effectively shorten the simulation time. However, the above methods still have the problems of low system security, high occupancy rate, and long response time.

Aiming at the above problems, this study contributes the design of a power intelligent customer service assistant decision-making system based on the Hadoop big data framework. By analyzing the Hadoop big data framework and combining data mining algorithms, the basic process of powerful intelligent customer service assistance decisionmaking is constructed. We analyze the overall and functional requirements of the system, design the overall architecture and application architecture of the system, and realize the design of power intelligent customer service assistant decision-making system. The proposed system is based on Hadoop big data framework according to the database E-R diagram and table structure. It has high system security, low occupancy, and short response time.

\section{Preliminaries of the Proposed System}

The proposed system is based on big data analytics tools that include Apache Hadoop for large-scale data processing and MapReduce framework, which is the key to the success of Apache Hadoop and other related frameworks. In this section, we are going to study these tools and frameworks in detail.

2.1. Hadoop Big Data Framework. Hadoop is a basic framework, which is used by many Internet companies to build distributed computing platforms. Without understanding the characteristics of the platform, users can easily develop and run the processing application platform. This platform has the characteristics of low cost and high expansion. It can effectively use the power of computer cluster to realize high-speed operation and large-capacity storage [6]. The Hadoop big data framework is shown in Figure 1.
The Hadoop big data framework is mainly composed of several components, namely, HDFS, Map Reduce, and Hive. Based on the HDFS distributed file storage system, the entire Hadoop big data framework is stored and managed. Based on Map Reduce distributed parallel operations, the entire Hadoop big data framework is calculated in parallel. Let Zoo keeper distributed applications as the basis to coordinate and serve the entire Hadoop big data framework. Data collection, processing, and conversion in the Hadoop big data framework are mainly implemented by Hbase, Hive, and Oozie, and Mahout is mainly used to implement data mining in the Hadoop big data framework. The Ganglia, Nagios, and other tools are mainly used to implement the monitoring and operation of the Hadoop big data framework. The Java language has the characteristics of powerful functions and simple operability, so this system is configured on most machines [7]. After the Hadoop cluster is developed through the Java language, it can be jointly run on multiple servers, which greatly reduces the calculation time of data.

A variety of slave nodes play an important role in Hadoop big data framework. Therefore, the Hadoop big data framework has the following advantages:

(1) Reliability: the Hadoop big data framework supports two master nodes and implements task scheduling for the framework. One master node is working, and the other master node is ready and keeps synchronized with its working master node in the framework. The working master node is mainly for external services, while the ready master node is mainly for internal services. When the working master node fails, you can quickly switch to the master node in the ready state and continue to complete relevant tasks.

(2) Scalability: Hadoop big data framework can enhance the data processing capacity of the framework by increasing the number of nodes. The scale of Hadoop big data framework nodes can be expanded to thousands of orders of magnitude, so as to effectively complete the automatic data allocation and calculation tasks of Hadoop big data framework.

(3) Ease of use: according to the open source characteristics of Hadoop big data framework, it can not only greatly reduce the software cost of this project, but also make Hadoop big data framework run on ordinary PCs with Linux system.

(4) High efficiency: the Hadoop big data framework can carry out real-time data interaction with HDFS distributed file storage system and can process data tasks in parallel with the help of map reduce distributed parallel operation. To a certain extent, the mobile data between various computing nodes of Hadoop big data framework is kept in dynamic balance, the processing speed is improved to the greatest extent, and it also reduces the working time of the subserver.

(5) High fault tolerance: Hadoop big data framework can save multiple copies of data to ensure that the 


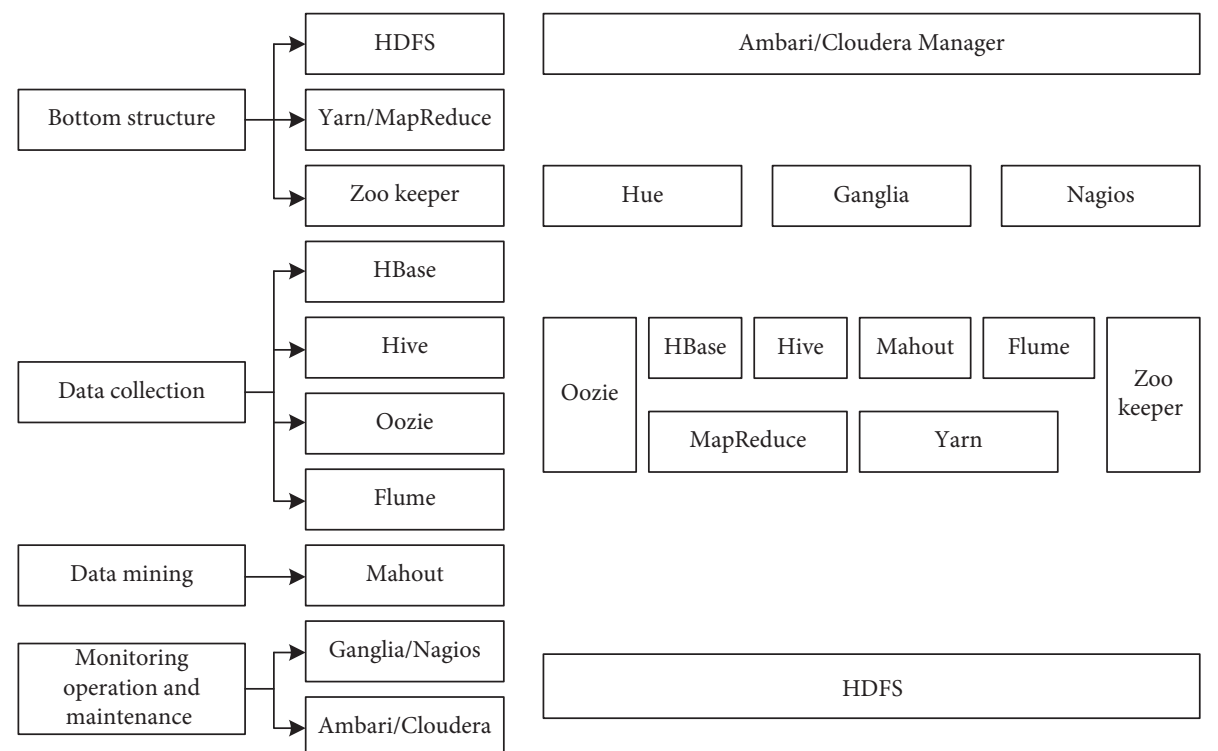

Figure 1: Hadoop big data framework.

function of data redistribution can be realized in case of abnormal tasks.

Hadoop has been widely used in the field of big data analysis, including business intelligence, web indexing, advertising, and log analysis. Hadoop big data framework has great advantages in data extraction, conversion, and loading because of its advantages of reliability, scalability, ease of use, high efficiency, and high fault tolerance. It can process data in batch and obtain results directly through operation.

2.2. HDFS Distributed File System. HDFS is a core component of Hadoop big data framework and belongs to the distributed file system of the framework. All storage node data in the framework are stored in it and can realize the data access function of high error tolerance and throughput [8]. The main storage method of HDFS adopts a master-slave node architecture, which consists of four types: client, a named node (Name Node), preparation node, and multiple data nodes (Data Node). The overall architecture of the HDFS distributed file system is shown in Figure 2.

As can be seen from Figure 2, through the interaction between the four types of master-slave nodes, the overall framework of HDFS distributed file system can run normally. The four types of master-slave node architecture have the following attributes and functions:

(1) Client: the main function is to split and store the original files in the form of data block. Obtain the location information of the original file according to the Name Node, read and write the original file according to the Data Node, and effectively manage the HDFS distributed file system of Hadoop big data framework through some instructions [9].

(2) Name Node:also known as master node. The main function is to maintain the information mapped by the block, process the information collected by the client, adopt the configuration replication strategy, and manage the HDFS namespace. The system maintains the file system tree and the Name Node of each file and records the location information of the block on the Data Node. When the system restarts, the recorded information will be reconstructed.

(3) Data Node:also known as slave node. The main function is to store the information received by the block. When the block reads and writes, it is equivalent to the case where the Name Node sends a command and the Data Node performs the actual operation.

(4) Secondary Name Node: it is mainly used to assist the Name Node and share the workload of the Name Node to a certain extent. In case of an emergency of Hadoop big data framework, it can help the Name Node recover its own functions.

2.3. MapReduce Programming Model. When Hadoop big data framework is used to process data, the data is stored in an HDFS and distributed in Hadoop big data framework, mainly through parallel processing of relevant data in a distributed manner. Map Reduce is a software framework that can be used to write applications. It uses parallel computing to process big data in the framework [10]. You can use Map Reduce to perform parallel operations on large-scale data and simplify the operation process. Even if you do not know much about parallel programming, you can write programs to run in the Hadoop big data framework. This is conducive to the Map Reduce programming model and effective scheduling of tasks on nodes, which improves the network bandwidth utilization of the entire Hadoop big data framework. The running framework of Map Reduce programming model is shown in Figure 3. 


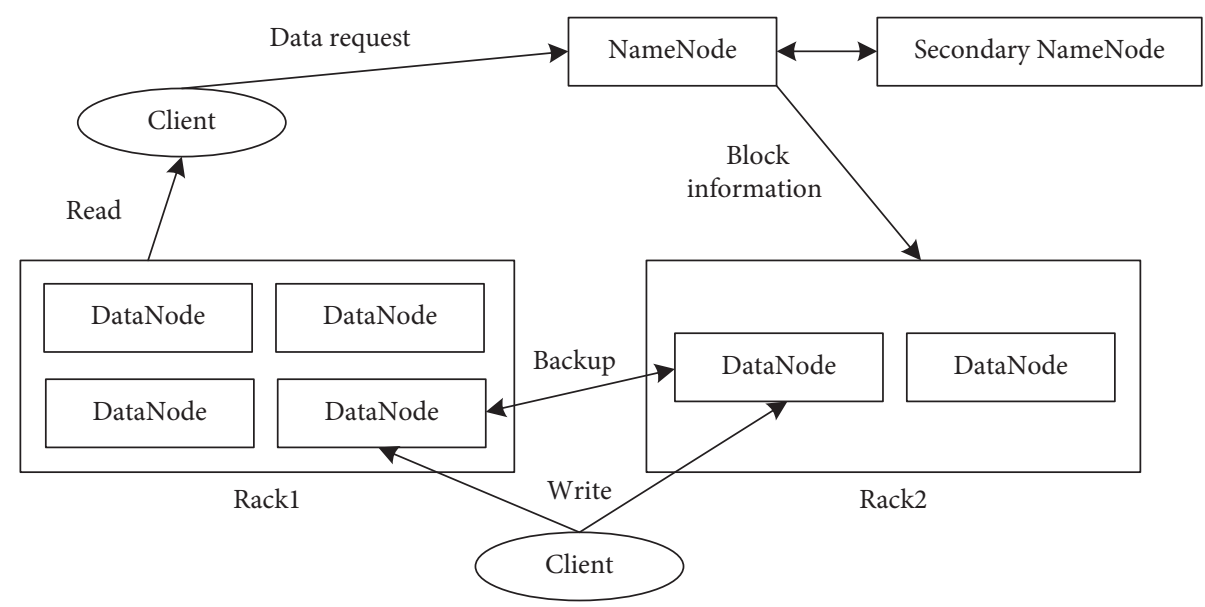

FIGURE 2: Overall architecture of HDFS distributed file system.

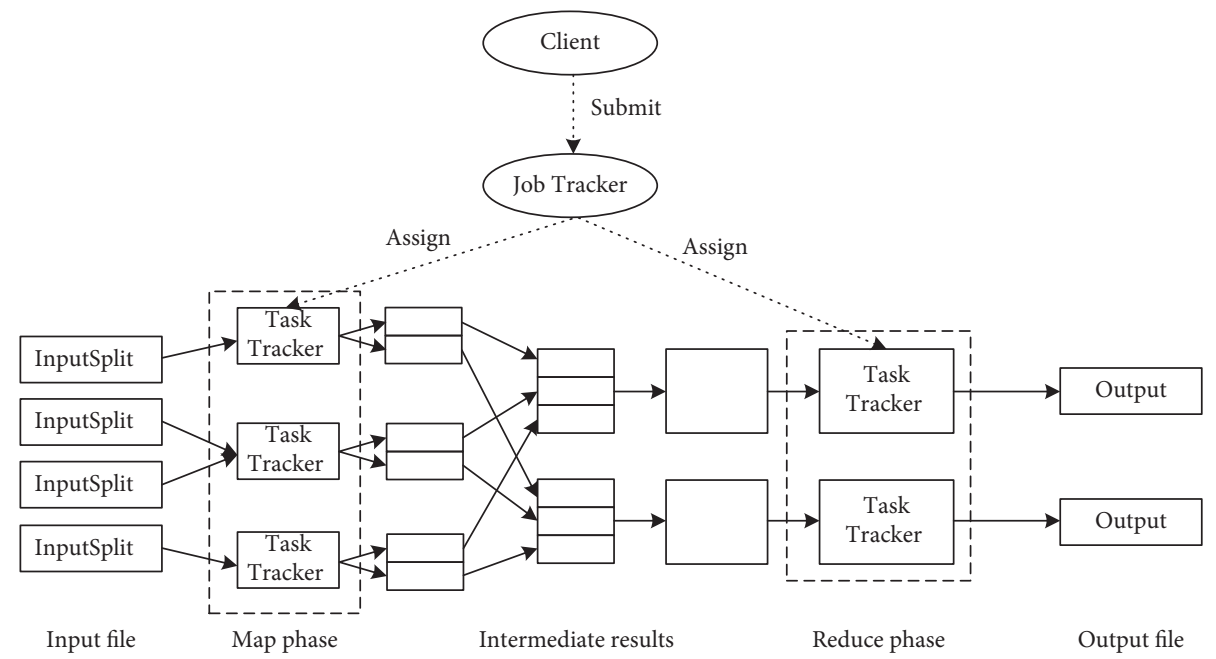

FIGURE 3: Map Reduce programming model framework.

It can be seen from Figure 3 that when a data task is submitted, the task manager will decompose the input data object into independent blocks. And hand it over to the Map stage for parallel processing to generate a corresponding intermediate process, and input this process to the Reduce stage to get the output result, and the final result is stored in the file system. The attribute functions of the four entities in the Map Reduce programming model framework of the Hadoop big data framework are as follows:

(1) Client: the main execution function is to write the Map Reduce program and configure and submit the Map Reduce program.

(2) Job Tracker: it is mainly responsible for real-time communication with Task Tracker; on this basis, it coordinates the initialization and assignment of task operations, schedules all tasks, and assigns different tasks on Task Tracker.

(3) Task Tracker: mainly maintain communication with Job Tracker, and process tasks that have been decomposed by Job Tracker. During task execution,
Task Tracker can have multiple tasks, while Job Tracker can only have one task.

(4) HDFS: it is mainly used to store working data, configuration information, share working files among other entities, and save the final results in HDFS.

2.4. Data Mining Algorithm. Data mining technology is a technology that combines traditional data analysis methods with complex algorithms for processing massive data [11]. The process of extracting meaningful data and information from incomplete, noisy, fuzzy, and random data is done.

At present, there are many algorithms in data mining. This paper uses the decision tree algorithm to mine the behavior data of power intelligent customer service channel interaction. In information theory and probability statistics, the degree of uncertainty of random variables can be expressed by entropy.

Suppose $Q$ is a discrete random variable, which has a finite value, and its $P\left(Q=q_{i}\right)=p_{i}, \quad i=1,2, \ldots, n$ is used 
to describe the probability distribution. Then, the entropy of the random variable $Q$ is defined as follows:

$$
W(Q)=-\sum_{i=1}^{n} p_{i} \log \left(p_{i}\right) .
$$

This is a random variable $(Q, Z)$, and its joint probability is $P\left(Q=q_{i}, Z=z_{j}\right)=p_{i j}, \quad i=1,2, \ldots, n, j=1,2, \ldots, m$.

When the random variable $Q$ is known, the conditional entropy $W(Z \mid Q)$ represents the uncertainty of the random variable $Z$, which is defined as the mathematical expectation of the conditional entropy on $Q$ as $Z$ under the conditions given by $Q$ :

$$
W(Z \mid Q)=\sum_{i=1}^{n} p_{i} W\left(Z \mid Q=q_{i}\right) .
$$

In decision trees, entropy can be used to measure the purity of a sample set [12]. Set the training data set $E$, the sample size is $|E|$, the sample is $k$ class $X_{i}, \quad i=1,2, \ldots, k$, the number of samples belonging to $X_{i}$ class is $\left|X_{i}\right|$, and a feature $M$ is $n$ different dispersion $\left\{m_{1}, m_{2}, \ldots, m_{n}\right\}$.

Based on the value of the feature $M, E$ is divided into $n$ subsets $R E_{1}, E_{2}, \ldots, E_{n}$ and the sample set belonging to $X_{i}$ in the notation subset $E_{j}$ is $E_{i j}$. So,

Information entropy:

$$
W(E)=-\sum_{i=1}^{k} \frac{\left|X_{i}\right|}{|E|} \log _{2} \frac{\left|X_{i}\right|}{|E|} .
$$
E.

The smaller the value, the higher the purity of the data set

Conditional entropy:

$$
\begin{aligned}
W(E \mid M) & =\sum_{j=1}^{n} \frac{\left|E_{j}\right|}{|E|} W\left(E_{j}\right) \\
& =-\sum_{j=1}^{n} \frac{\left|E_{j}\right|}{|E|} \sum_{i=1}^{k} \frac{\left|E_{i j}\right|}{\left|E_{j}\right|} \log _{2} \frac{\left|E_{i j}\right|}{\left|E_{j}\right|} .
\end{aligned}
$$

\section{Design of the Auxiliary Decision-Making System}

In this section, we are going to discuss the requirements and design of the auxiliary decision-making system based on Hadoop and MapReduce frameworks.

3.1. System Requirements Analysis. The designed power intelligent customer service assistant decision-making system shall provide a comprehensive technical support and data service platform for the operation monitoring and analysis, label management system, support service, and system management of power intelligent customer service channel of a local power supply bureau.

(1) Construction of multisource data processing capacity: around the functional application construction requirements of electric energy big data system, connect with the star ring platform, build data acquisition channels, and build internal and external data application processing capacity, so as to lay a foundation for the development of service applications.

(2) Realize the operation analysis of power intelligent customer service channel: the operation and business handling indicators of each power intelligent customer service channel are monitored through Hadoop big data framework, and the comprehensive operation of power intelligent customer service channel is analyzed.

(3) Build the power intelligent customer service label management system: the power intelligent customer service behavior feature library is established according to the characteristics of users. The characteristics of typical power intelligent customer service behavior are identified, and the types of power intelligent customer service are divided in more detail to realize automatic identification.

3.1.1. Functional Requirements Analysis. It is assumed that the designed power intelligent customer service auxiliary decision-making system has the functions of power intelligent customer service channel monitoring and analysis and label system management.

(1) Operation analysis of power intelligent customer service channel: unified channel classification specifications, interface specifications and update mechanism are formulated for power intelligent customer service. A unified channel information pool is built, the data application requirements are analyzed, and channel information is quickly retrieved by using big data technology. The electric intelligent customer service channel record can obtain user interaction information and typical customer characteristics and build a preference model through the above information. According to the access of power intelligent customer service channel to users, the construction of a personalized recommendation algorithm is completed. Develop an all-weather monitoring and unified management platform for the power intelligent customer service channel to monitor the utilization of power intelligent customer service channel resources and the development of channel business in real time. An analysis model of abnormal causes of power intelligent customer service channel is proposed, which can be used as a reference for channel accident handling.

(2) Power intelligent customer service label system management: the main function is to screen and group customers according to electronic labels, build a power intelligent customer service behavior feature library through the obtained user characteristics, realize the data analysis of customer behavior, and 
configure electronic labels according to the characteristics. Using the methods of feature extraction, cluster analysis and pattern recognition, the typical power intelligent customer service behavior is identified, and the types of power intelligent customer service are divided more carefully to realize automatic identification. By identifying the combination of electric equipment, the behavior and demand of power intelligent customer service are described and analyzed in more detail, and the temporal and spatial distribution characteristics are constructed.

3.2. Overall System Design. The overall architecture of power intelligent customer service assistant decision-making system based on Hadoop big data framework is mainly composed of infrastructure layer, basic software layer, customer service support layer, and application layer. The overall architecture of power intelligent customer service assistant decision-making system is shown in Figure 4.

(1) Basic architecture layer: it is composed of infrastructure physical servers, storage devices, and resource libraries.

(2) Basic software layer: it is composed of data warehouse platform, software, and middleware. The big data warehouse platform provides basic functions such as distributed file storage, resource management, integration, and storage.

(3) Customer service support layer: power intelligent customer service support for data retrieval, integration, query, and mining by big data packets.

(4) Application layer: focus on the integration, management, and application of internal and external data of power energy, further plan and sort out the application functions, and realize advanced applications such as operation analysis of power intelligent customer service channel and label system management, so as to realize the unified management and analysis of power intelligent customer service.

3.2.1. System Application Architecture Design. The application architecture of power intelligent customer service assistant decision system based on Hadoop big data framework mainly includes data management, basic application, advanced application, decision center, and system management. The application architecture of power intelligent customer service assistant decision system is shown in Figure 5.

(1) Data management: its main function is to manage data access. The access methods include interface to internal access, multiple server interfaces to external data, service registration, unified management of interface services, introduction of comprehensive evaluation standards of data quality, and data cleaning and modification [13]. To realize data integration, we need to collect, sort out, and clean the data from different data sources, convert the integrated data, load it into new data sources, and uniformly create a data integration view that is easy for data consumers to observe.

(2) Basic application: mainly through report statistics, data mining, data prediction, and other service applications, the basic application construction of the application framework is carried out to build a platform for service capability, service capability automation, and data application autonomy.

(3) Advanced applications: mainly through the big data warehouse platform, basic application capabilities, internal and external power big data, analysis of customer service channels, management of power customer file label database and power efficiency, etc.

(4) System management: it mainly unifies the system platform management functions such as system permission management, log management, user management, and infrastructure management.

\subsection{System Function Design}

(1) Business process of power intelligent customer service channel operation analysis: the main business process of power intelligent customer service channel operation analysis is shown in Figure 6.

(2) Panoramic real-time monitoring of power intelligent customer service channel: obtain the application requirements by collecting the data of power intelligent customer service channel, view the panoramic information of power intelligent customer service channel by using big data technology, carry out realtime monitoring of the operation of each power intelligent customer service channel, analyze the business handling of power intelligent customer service channel in real time, and monitor the whole process of work order circulation. Assist business personnel to fully understand the operation status of power intelligent customer service channel, quickly explore business problems, and provide decisionmaking information for power intelligent customer service channel construction, channel optimization, and channel resource allocation.

(3) Comprehensive analysis of power intelligent customer service channel monitoring: monitor the operation of power intelligent customer service channel, work order handling and work order flow of functional departments, and conduct real-time online monitoring of various power intelligent customer service channels or overall business indicators [14]. The behavior data of power intelligent customer service channel interaction is mined, the demand preference and channel preference of power intelligent customer service are analyzed, and the power intelligent customer service channel 


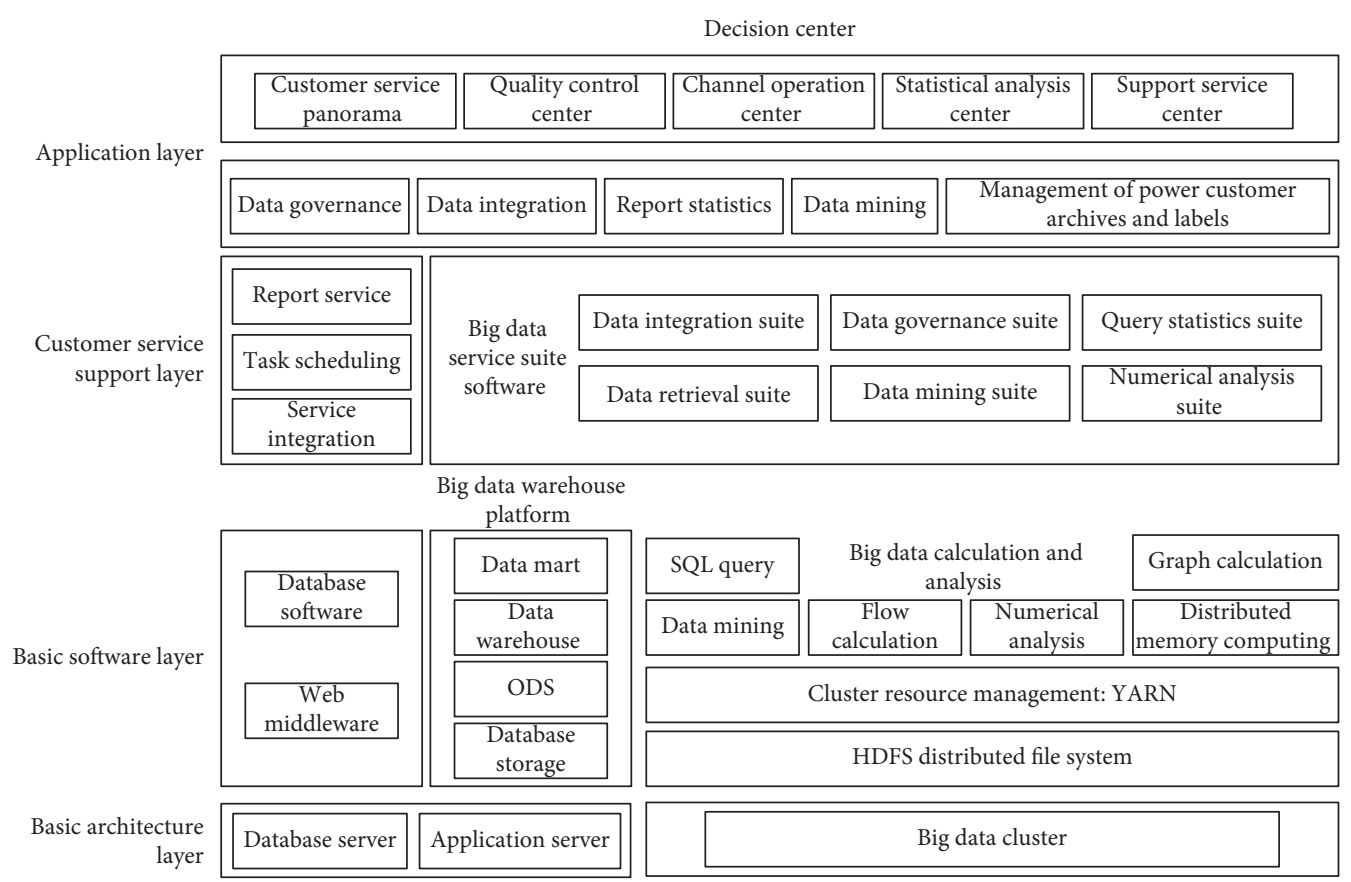

FIGURE 4: Overall architecture of power intelligent customer service assistant decision system.

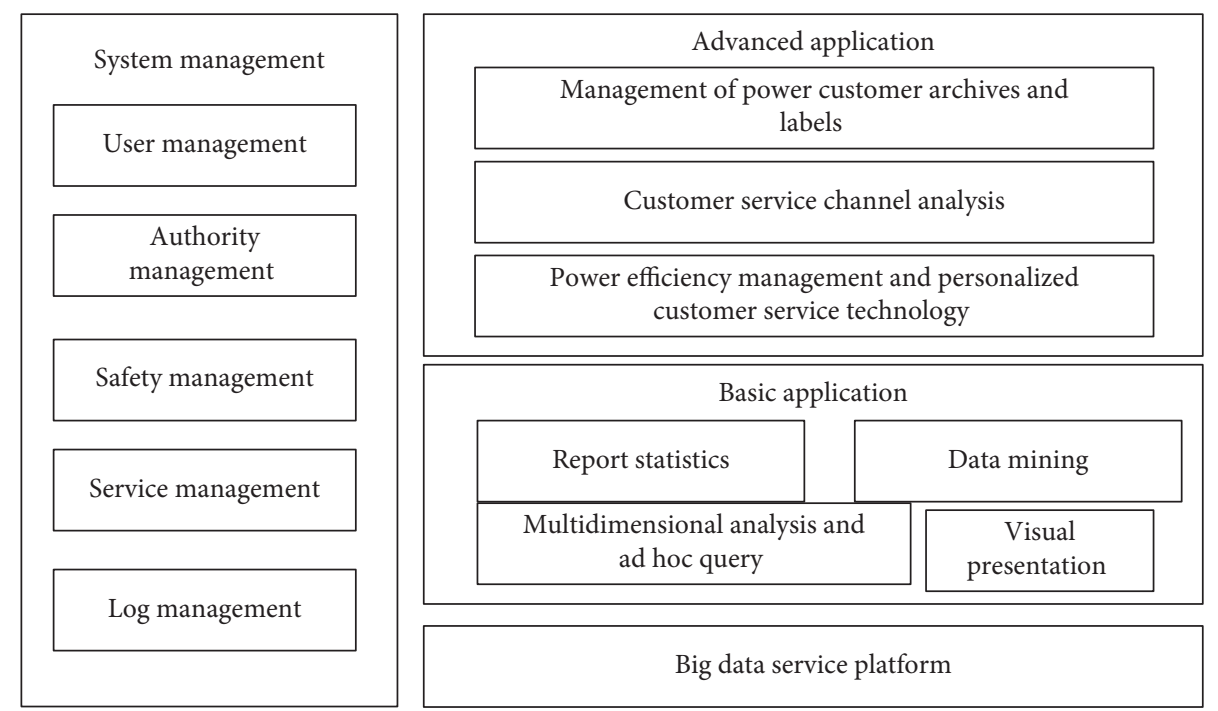

FIGURE 5: Application architecture of power intelligent customer service assistant decision-making system.

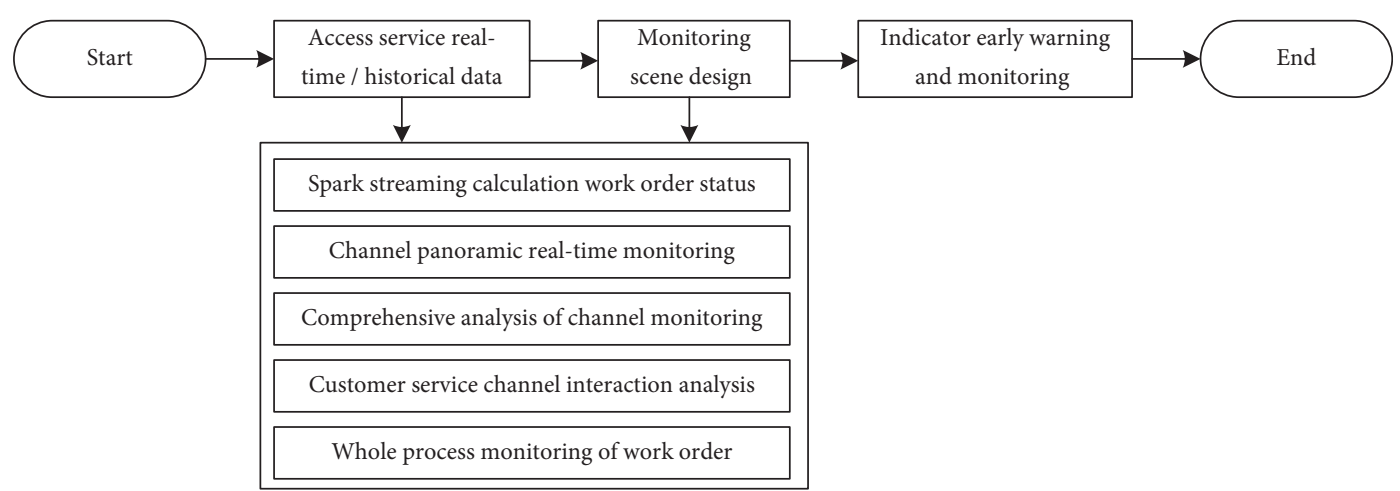

FIGURE 6: Business flow chart of operation analysis of electric power intelligent customer service channel. 
preference model is proposed to match the demand strategy of power intelligent customer service.

(4) Whole process monitoring of electric power intelligent customer service work order: monitor the channel, business type, regional work order status, time limit distribution, and work order status of electric power intelligent customer service channel and functional departments during the circulation process, conduct on-the-spot monitoring, count the details of the screened work orders, and send urging SMS to the screened abnormal work orders.

3.3.1. Power Intelligent Customer Service Label System Management. Tag processing for users is conducive to the use of data mining methods such as feature processing, cluster analysis, and pattern recognition, so as to realize the identification of typical power intelligent customer service behavior characteristics. The types of electric power intelligent customer service are divided more carefully to realize automatic identification, more detailed description, and analysis of electric power intelligent customer service behavior and demand, and the characteristics of temporal and spatial distribution are constructed. The big data retrieval method is used to explore the heterogeneous data hiding mode of user preference. Through various forms of feedback information such as customer complaint and consultation, the customer classification, and multidimensional image display of power intelligent customer service demand can be carried out. Through the management of electric power intelligent customer service archives and labels, provide basic tools, including data mining and analysis, data visualization, intelligent report, data export, and other common functions in the process of data application, so as to achieve the comprehensive support of the platform's service capability, automation, and independent data application.

(1) Business process of power intelligent customer service label system management: the main business process of customer label system management is shown in Figure 7.

(2) New user tag: by using the process method, the user can generate the query statement corresponding to the configuration after a series of drag and drop operations on the page as required. After execution, the system background will run the query statement in the Hadoop big data framework and provide feedback about the query results to the foreground, which will display the relevant query results.

(3) User defined label display: data integration process management is to add, delete, modify, and query existing data processes in the system. If the process status is not being edited, it cannot be edited.

(4) Power intelligent customer service label system management: manage the labels formed by data mining and custom labels of power intelligent customer service, including query, addition, deletion, and other operations.
(5) Intelligent report: the intelligent analysis report is to reflect the function of intelligent blank filling. By introducing the form of word text report, the paragraph text or table number and other information in the text report can be introduced for flexible presentation and analysis [15].

3.4. Database Design. Database design is one of the important parts of the system. Use the data in the database memory integrated management system, including adding, deleting, modifying, and querying functions. By analyzing each actual business, sort out the main data generated and required by the operation business of power intelligent customer service channel. These data mainly include master data and business data:

(1) Master data refers to customer file, equipment account, and other file data.

(2) Business data refers to various business data generated during the specific business operation of power intelligent customer service, such as measurement data models such as electricity charge, power load, and power line loss, and then form a specific database entity table according to the formed data models and their attributes.

3.4.1. Database E-R Diagram Design. The core database table structure of the database is designed and analyzed in detail. The E-R diagram of the designed database is shown in Figure 8.

3.4.2. Database Table Structure Design. According to the designed database E-R diagram, the main data tables and business data tables of the system are defined as follows:

(1) Power user file data table: mainly composed of power user information CUSTOMER, power access information C_POWER, power address C_ADDRESS, contact person CONTACT, running meter reading RUN_METERNUM, and running meter file RUN_METER. Among them, the electricity access information is user power information, which mainly includes the main attributes such as power source type, power source nature, line identification, station area identification, and property rights demarcation point.

(2) Power smart customer service data table: mainly composed of business hall business SERVICE_HALL, power smart customer service website business acceptance volume SERVICE_NETWORK, power smart customer service type SERVICE_TYPE, and customer appeal ERVICE_APPEAL.

Through the above steps, the design of power intelligent customer service assistant decision system based on Hadoop big data framework is realized. 


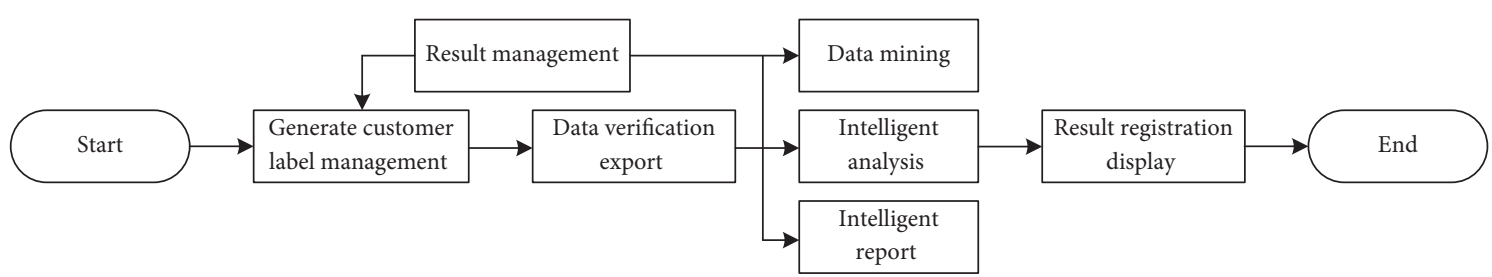

Figure 7: Business flow chart of the management of the electric power intelligent customer service label system.

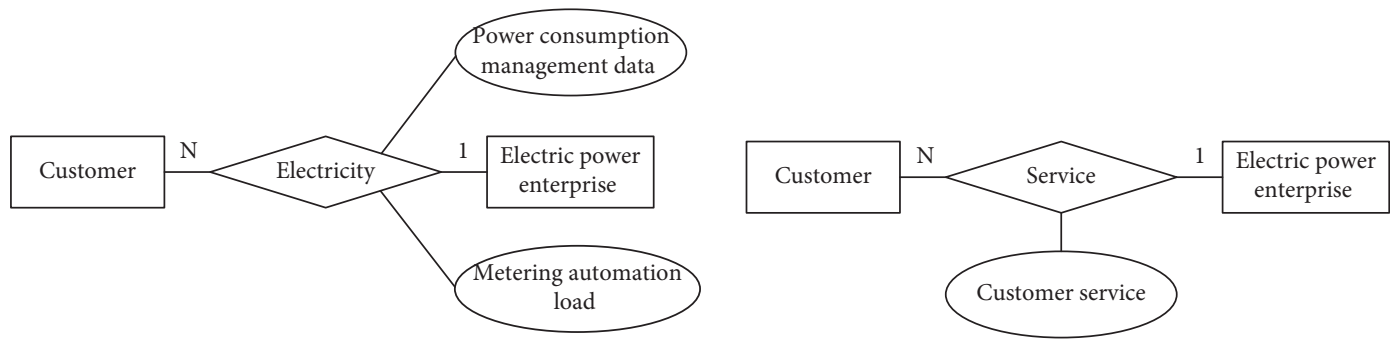

Figure 8: E-R diagram of design database.

\section{Experimental Environment Setup}

In order to test the effectiveness of the power intelligent customer service assistant decision-making system based on Hadoop big data framework, by analyzing the functions of the designed module, according to the business process, the designed power intelligent customer service assistant decision-making system is systematically tested with perfect test tools to verify its effectiveness. Through complete testing techniques and methods, unit testing, iterative testing, integration testing, and system testing are carried out, respectively, and the test results are analyzed to verify the safety and functionality of the system. Using HP LoadRunner to test system performance can ensure the efficiency and quality of the test. In the process of system testing, it is necessary to test according to the standard specification process of CMMI L5. Its professional test process includes test scheme formulation, test case design, test execution, test result evaluation, and defect tracking management, which can ensure the quality of software products. The method of reference [4], the method of reference [5], and the proposed method are compared to verify the performance of the proposed method.

4.1. System Safety Test Results. In order to test the security of the system designed by the proposed method, the number of bugs in the system is taken as the evaluation index. The smaller the number of bugs in the system, indicating the fewer system vulnerabilities, the higher the security. By comparing the method of reference [4], the method of reference [5], and the proposed methods, the number of bugs in the systems of different methods is obtained, and the comparison results are shown in Figure 9.

According to Figure 9, the number of bugs in the systems of the three methods is different in different test stages of the system. Among them, in the system unit test stage, the number of bugs in the system of the method of reference [4],

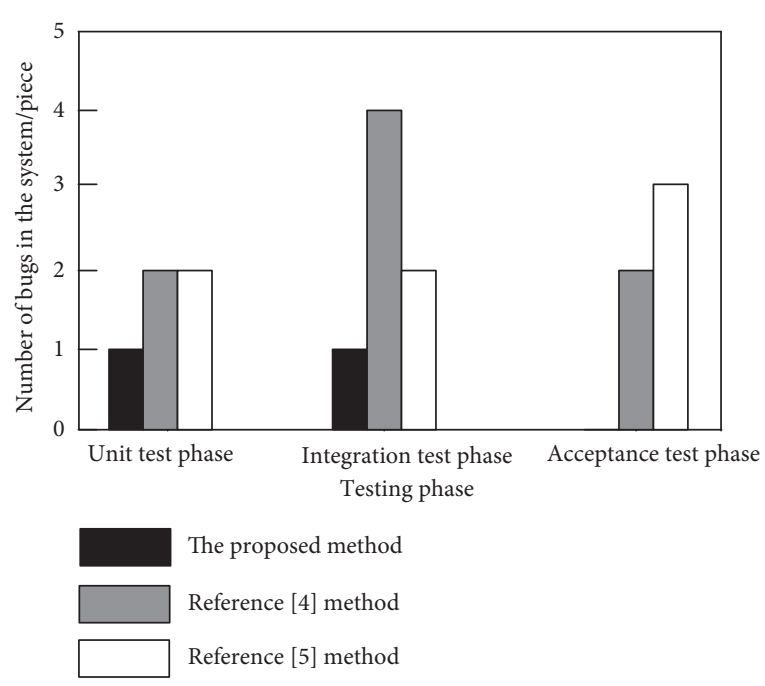

Figure 9: Comparison results of the number of bugs in systems with different methods.

the method of reference [5], and the proposed method is 2, 2, and 1 , respectively. In the system integration test stage, the number of bugs in the system of the method of reference [4], the method of reference [5], and the proposed method is 4, 2, and 1 , respectively. In the system acceptance test stage, the number of bugs in the system of the method of reference [4], the method of reference [5], and the proposed method is 2, 3, and 0 , respectively. It can be seen that, compared with the method of reference [4] and the method of reference [5], the number of bugs in the system of the proposed method is smaller, indicating that the system has fewer vulnerabilities and higher security.

4.2. System Occupancy Test Results. In order to verify the occupancy of the system designed by the proposed method, the CPU utilization is taken as the evaluation index. The 


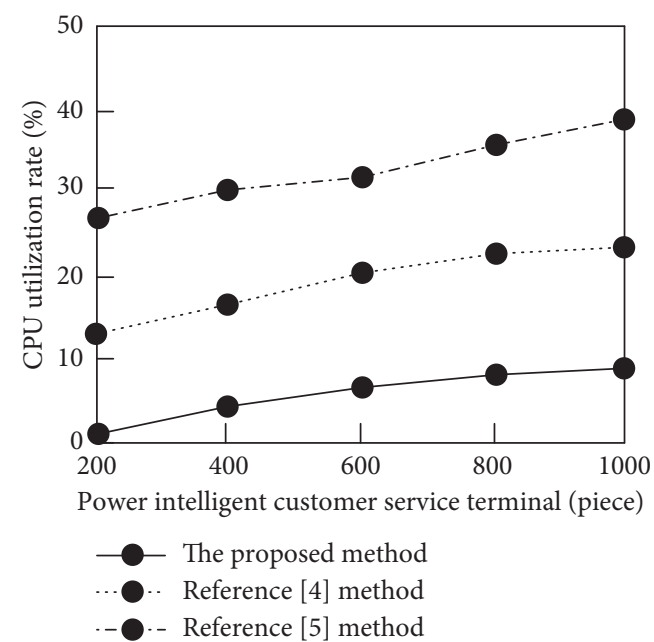

Figure 10: Comparison results of system occupancy rate of different methods.

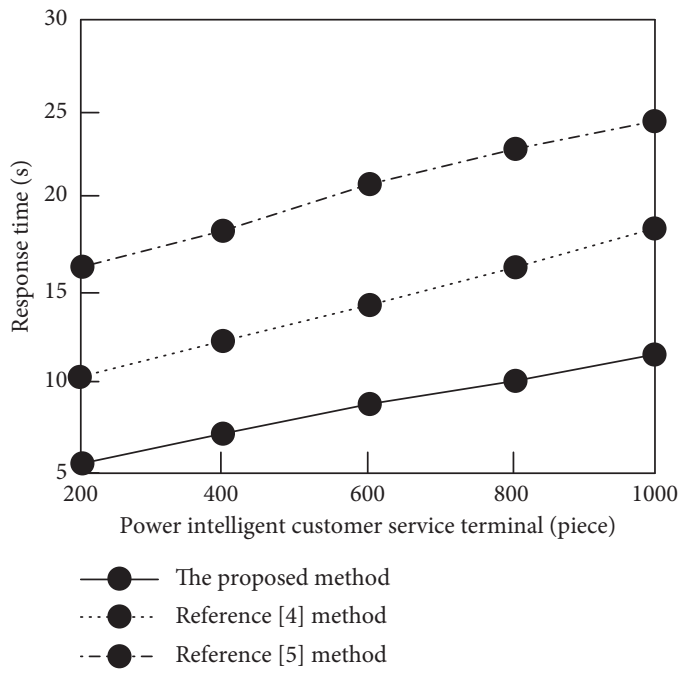

Figure 11: Comparison results of system response time of different methods.

higher the CPU utilization, the higher the occupancy of the system. By comparing the method of reference [4], the method of reference [5], and the proposed methods, the system occupancy comparison results of different methods are obtained, as shown in Figure 10.

As can be seen from Figure 10, with the increase of the number of power intelligent customer servers, the system CPU utilization of different methods increases. When the number of system power intelligent customer servers reaches 1000, the system CPU utilization rate of the method of reference [4] is $23.5 \%$, the system CPU utilization rate of the method of reference [5] is $39.7 \%$, and the system CPU utilization rate of the proposed method is $9.2 \%$. It can be seen that, compared with the method of reference [4] and the method of reference [5], the system CPU utilization rate of the proposed method is low, indicating that the system occupancy rate is low.
4.3. System Response Time Test Results. On this basis, the response time of the system designed by the proposed method is further verified. The method of reference [4], the method of reference [5], and the proposed method are compared, respectively, and the system response time test results of different methods are obtained, as shown in Figure 11 .

It can be seen from Figure 11 that, with the increase of the number of power intelligent customer servers, the system response time of different methods increases. When the number of power intelligent customer servers is 1000, the system response time of the method of reference [4] is $18.5 \mathrm{~s}$, the system response time of the method of reference [5] is $24.5 \mathrm{~s}$, and the system response time of the proposed method is only $12.2 \mathrm{~s}$. It can be seen that the system response time of the proposed method is shorter than that of the method of reference [4] and the method of reference [5].

\section{Conclusion}

The power intelligent customer service assistant decision system based on Hadoop big data framework designed in this paper gives full play to the advantages of Hadoop big data framework. Its power intelligent customer service assistant decision system has high security and low occupancy rate and can effectively shorten the system response time. However, in the power intelligent customer service assistant decision-making system, it is necessary to further improve the system functions, effectively expand the application functions of different modules, and deeply explore the connection relationship between different modules. Therefore, in the next research, it is necessary to further build the system function, improve and adjust the system operation process, and realize a more comprehensive power intelligent customer service auxiliary decision-making system. In future, we plan to extend our work by testing more big data analytics tools such as Apache Spark to improve the performance of the proposed system and reduce the response time.

\section{Data Availability}

The data used to support the findings of this study are included within the article.

\section{Conflicts of Interest}

The authors declare that they have no conflicts of interest.

\section{References}

[1] C. B. Wu, P. B. Guan, L. N. Zhong et al., "An optimized lowcarbon production planning model for power industry in coal-dependent regions-a case study of Shandong, China," Energy, vol. 192, Article ID 116636, 2020.

[2] H.-H. Yu, K.-H. Chang, H.-W. Hsu, and R. Cuckler, "A monte carlo simulation-based decision support system for reliability analysis of taiwan's power system: framework and empirical study," Energy, vol. 178, pp. 252-262, 2019. 
[3] J. Gao, Z. Ma, and F. Guo, "The influence of demand response on wind-integrated power system considering participation of the demand side," Energy, vol. 178, pp. 723-738, 2019.

[4] T. B. de Oliveira Barreto, P. R. Pinheiro, and C. F. G. Silva, "The multicriteria model support to decision in the evaluation of service quality in customer service," Advances in Intelligent Systems and Computing, pp. 158-167, 2018.

[5] T. Xiao, Y. Zou, Y. Xia, W. Tong, and J. Wang, "Design and tests of a super real-time simulation-based power system realtime decision-making emergency control system," IET Generation, Transmission \& Distribution, vol. 14, no. 9, pp. 6812-6819, 2020.

[6] A. K. Javanmardi, S. H. Yaghoubyan, K. Bagherifard, S. Nejatian, and H. Parvin, "A unit-based, cost-efficient scheduler for heterogeneous Hadoop systems," The Journal of Supercomputing, vol. 77, no. 4, 2021.

[7] D. Silva, J. P. Silva, G. Santos, R. Terra, and M. T. O. Valente, "RefDiff 2.0: a multi-language refactoring detection tool," IEEE Transactions on Software Engineering, vol. 47, Article ID 2968072, 2020.

[8] W. Xu, X. Zhao, B. Lao, and C. Nong, "Enhancing HDFS with a full-text search system for massive small files," The Journal of Supercomputing, vol. 77, no. 4, pp. 1-22, 2021.

[9] D. Chen, R. Zhang, and H. S. Catla, "An open source project for tuning and analyzing MapReduce performance on Hadoop and Spark," IEEE Software, vol. 39, Article ID 3044598, 2020.

[10] A. Addisie and V. Bertacco, "Collaborative accelerators for streamlining MapReduce on scale-up machines with incremental data aggregation," IEEE Transactions on Computers, vol. 69 , no. 8, pp. 1233-1247, 2020.

[11] L. Huang, "Design of an IoT DDoS attack prediction system based on data mining technology," The Journal of Supercomputing, no. 8, pp. 1-23, 2021.

[12] M. Shaik, S. K. Yadav, and A. G. Shaik, "An EMD and decision tree based protection algorithm for solar PV integrated radial distribution system," IEEE Transactions on Industry Applications, vol. 57, no. 3, Article ID 3058618, 2021.

[13] N. Guo, Y. Shen, Y. Han, and P. Li, "Simulation of dynamic cleanup of inferior user data under big data analysis," Computer Simulation, vol. 36, no. 2, pp. 362-366, 2019.

[14] M. Majumder, A. K. Saha, and P. Majumder, "Real time monitoring of power production in modular hydro power plant: most significant parameter approach," Environment, Development and Sustainability, vol. 22, no. 2, pp. 4025-4042, 2020.

[15] Z. Liang, D. Pan, and R. Xu, "Knowledge representation framework of accounting event in corpus-based financial report text," Cluster Computing, vol. 22, no. 4, pp. 9335-9346, 2019. 